\title{
Growth of $\mathrm{Li}_{x} \mathrm{La}_{y} \mathrm{Sr}_{z} \mathrm{MnO}_{3}$ thin films by pulsed laser deposition: complex relation between thin film composition and deposition parameters
}

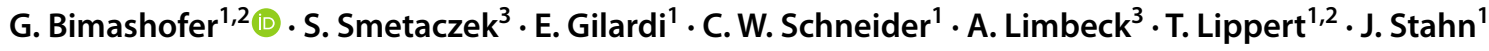

Received: 16 February 2021 / Accepted: 10 April 2021 / Published online: 31 May 2021

(c) The Author(s) 2021

\begin{abstract}
$\mathrm{Li}_{x} \mathrm{La}_{y} \mathrm{Sr}_{z} \mathrm{MnO}_{3}$ thin films of various compositions $(x, y, z)$ have been grown using pulsed laser deposition. The compositions of the films have been studied as a function of deposition temperature, target-to-substrate distance and deposition pressure with respect to different cation ratios of the targets by inductively coupled plasma mass spectrometry. When growing multielemental oxide thin films containing lithium (with its large mass difference to other elements), lithium loss is most probably inevitable. But the desired thin film composition can be achieved by selecting specific growth conditions and different target compositions. The experiments also elucidate some of the mechanisms behind the incongruent lithium transfer from the targets to thin films.
\end{abstract}

Keywords Thin film $\cdot$ Pulsed laser deposition $\cdot$ Complex oxide $\cdot$ Lithium

\section{Introduction}

This project is funded by the Swiss National Science Foundation under Grant: 200021_169704 and carried out at Paul Scherrer Institute, Switzerland.

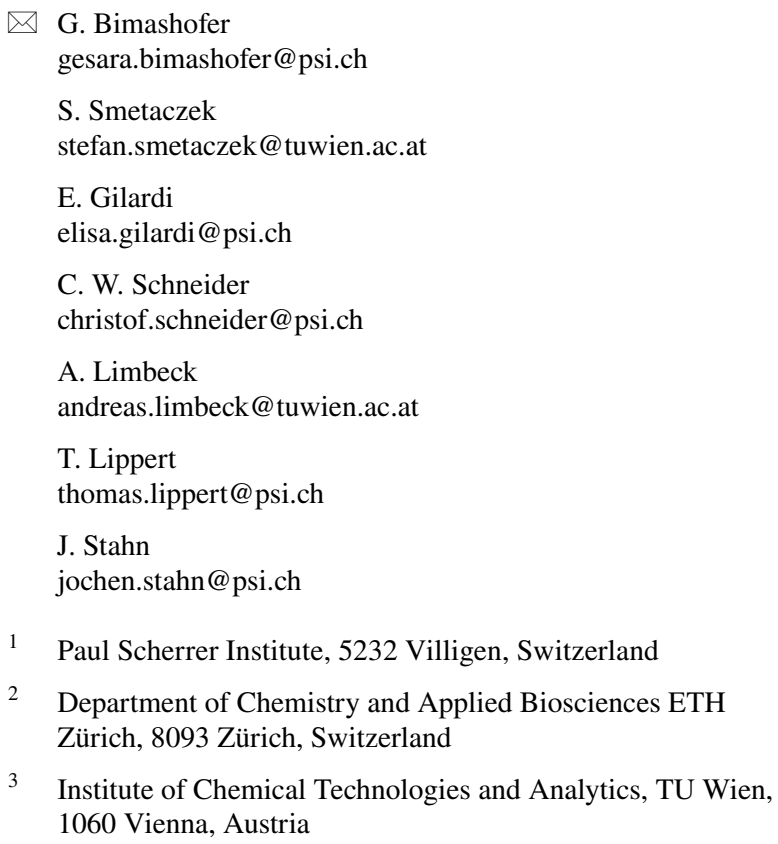

3 Institute of Chemical Technologies and Analytics, TU Wien, 1060 Vienna, Austria

The general interest to fabricate lithium containing thin films has been growing since they can be used and integrated in micro-batteries utilised as power sources for nano-devices like smart cards, implantable medical devices and microsensors. [1,2] For these applications, it is necessary to grow high-quality thin films (with respect to crystallinity, composition, etc.) containing light and reactive elements like $\mathrm{Li}$ and heavier elements such as $\mathrm{Co}, \mathrm{Mn}, \mathrm{Ni}$. [3] The increased activity in developing all solid-state lithium batteries has therefore sparked extensive research to produce and characterise lithium containing films including oxides. [4, 5]

Our future aim is to electrochemically control the magnetic properties of lithiated $\mathrm{La}_{1-x} \mathrm{Sr}_{x} \mathrm{MnO}_{3}$ (LSMO) to help disentangle the relationship between charge ordering and magnetism in manganites. The magnetic properties of LSMO are a function of the doping level and hence of the Mn oxidation state. [6] Introducing Li into the overall composition will contribute to the net valence and therefore provides means to study properties of magnetic phase boundaries in systems like $\mathrm{La}_{1-x} \mathrm{Sr}_{x} \mathrm{MnO}_{3}$ with $x \approx 0.5$, where this material exhibits a transition between ferro- and paramagnetism at room temperature. [7]

Pulsed laser deposition (PLD) is widely used to fabricate thin oxide films with a complex composition because 
of the often described congruent material transfer. [8] Conversely, it has been shown that a congruent transfer is not always the case and depends on a wide range of parameters. [9] One very important parameter is the mass ratio of the involved species with a large ratio leading to distinct composition deficiencies. [10] Several studies exhibited the influence of the used background gas on the composition of the grown film. [8, 10-14] E.g. Ohnishi et al. [15] proved that $\mathrm{LiCoO}_{2}$ films become lithium deficient at higher oxygen background pressures. For $\mathrm{LiMn}_{2} \mathrm{O}_{4}$, Dumont et al. [16] also showed that there are deviations in composition for high background pressures and therefore have a strong influence on chemical and physical properties. From these observations, one can conclude that the fabrication of materials containing a mixture of light and heavy elements with PLD leads to deviations in the films composition at certain pressures.

It is imperative for the magnetic properties of the material to grow highly crystalline LSM/LiLSM thin films with the corresponding oxygen stoichiometry.[17, 18] Therefore, adversely to the warnings in literature, high oxygen background pressures and post annealing are used to grow the films during the conducted experiments.

The focus of this study is to grow lithiated $\mathrm{La}_{1-x} \mathrm{Sr}_{x} \mathrm{MnO}_{3}$ (LiLSM) films using PLD from ceramic targets with variations in the A-site cation stoichiometries and to establish sample growth parameters for lithiated LSM films, namely $\mathrm{Li}_{0.1} \mathrm{La}_{0.5} \mathrm{Sr}_{0.4} \mathrm{MnO}_{3}$ with oxygen as background gas. First, a variety of target compositions was used and process parameters such as background pressure, laser fluence, target-tosubstrate distance and temperature were kept constant. In a second series of experiments, two target distances were tested for one target composition $(4 \mathrm{~cm}$ and $7 \mathrm{~cm}$ ). Finally, the influence of the deposition temperature upon Li transfer was studied complemented by depositions at room temperature.

PLD was chosen because it allows a high degree of flexibility which might be necessary when trying to optimise growth parameters for multi-element oxides. It also allows the growth of thin films with compositions that are non-existent in bulk by tuning the process parameters. [19] As the method exhibits a high degree of freedom when it comes to growth conditions it is also challenging to find the best conditions for the desired thin film. In this specific case, these experiments were therefore helpful to elucidate some of the dynamics leading to lithium loss during the PLD process when growing multielement oxide thin films with a mix of very light and heavy cations.

\section{Experimental details}

\subsection{Target preparation}

Various LiLSM PLD-targets were produced using the solid-state route. For which $\mathrm{La}_{2} \mathrm{O}_{3}$ (Sigma Aldrich 99.999\%), $\mathrm{SrCO}_{3}$ (Alfa Aesar 99.99\%), $\mathrm{MnO}_{2}$ (Sigma Aldrich 99\%) powders were used in stoichiometric amounts and $\mathrm{Li}_{2} \mathrm{CO}_{3}$ (Alfa Aesar 99\%) was applied in excess $(10 \%, 30 \%, 200 \%, 400 \%)$, to compensate for lithium loss during sintering. [20] The mixtures were pressed into pellets, sintered at $1473 \mathrm{~K}$ and reground twice. The target-IDs ( $\mathrm{T}$ for target) correspond to the remaining lithium content determined by inductively coupled plasma mass spectrometry (ICP-MS) in the target after sintering (see Table 1).

\subsection{Thin film preparation}

After pre-ablation of the targets, LiLSM thin films were deposited on $5 \times 5 \times 1 \mathrm{~mm}^{3}$ Yttria-stabilised $\mathrm{ZrO}_{2}$ substrates (Crystec). The depositions were performed using an excimer laser $(\lambda=248 \mathrm{~nm})$, ablating four of the ceramic pellets mentioned above, namely T-Li-0.33, T-Li-0.13, T-Li-0.08, T-Li-0.06. The spot size of the laser on target was $1 \mathrm{~mm}^{2}$ and the fluence was $1.5 \mathrm{~J} / \mathrm{cm}^{2}$. A laser frequency of $2 \mathrm{~Hz}$ and a substrate temperature of $923 \mathrm{~K}$ were used, except for an additional deposition of T-Li-0.06 at $823 \mathrm{~K}$. The oxygen background pressure was set between 10 and $50 \mathrm{~Pa}$. This pressure range was chosen to insure the oxygen stoichiometry, and therefore the appropriate $\mathrm{Mn}$ oxidation state [21] in the films. The distance from target to substrate was set to $4 \mathrm{~cm}$ for most films, one additional ablation sequence with T-Li-0.13 was done at $7 \mathrm{~cm}$ with a deposition time of 30 minutes for each film. The subsequent annealing in $\mathrm{O}_{2}\left(10^{4} \mathrm{~Pa}\right)$ was done at $723 \mathrm{~K}$. The film-IDs ( $\mathrm{F}$ for film) correspond to the lithium content in the target and the lithium content in the film (Table 2).

Table 1 Overview of the as prepared targets, including the lithium content per formula unit (pfu) (relative to $\mathrm{Mn}=1$ ) before and after sintering

\begin{tabular}{lll}
\hline Target-ID & \multicolumn{2}{l}{ Li content $[\mathrm{pfu}] \pm \sigma$} \\
\cline { 2 - 3 } & Before/ & After sintering \\
\hline T-Li-0.33 & 0.40 & $0.33 \pm 0.0(06)$ \\
T-Li-0.13 & 0.20 & $0.13 \pm 0.00(3)$ \\
T-Li-0.08 & 0.13 & $0.08 \pm 0.01(0)$ \\
T-Li-0.06 & 0.10 & $0.06 \pm 0.00(1)$ \\
\hline
\end{tabular}


Table 2 Variation of the process parameters such as temperature $T$, distance $D$, pressure $P$ during PLD and the lithium content (pfu) (again relative to $\mathrm{Mn}=1$ ) in the target and resulting thin film

\section{Characterisation}

The compositions of the synthesised targets as well as the compositions of the thin films ( 3 from each target) were determined by ICP-MS. [22] A quadrupole instrument (Thermo iCAP Qc, ThermoFisher Scientific, Germany) was used for all measurements. Sample digestion was achieved using hot aqua regia $\left(80^{\circ} \mathrm{C}, 3 \mathrm{~h}\right)$. For signal quantification, conventional external calibration using aqueous standards was performed and internal standardisation (1 ng/g indium) was applied. All standards were prepared from certified single element ICPstandard solutions (Certipur, Merck, Germany). Prior to each experiment, instrument settings were optimised for maximum indium-115 signal using a tuning solution provided by the manufacturer of the instrument.

For the crystallographic measurements of the target powders, a high-resolution X-ray powder diffractometer (Bruker D8) with $\mathrm{Cu} \mathrm{K} \alpha$-radiation $(\lambda=1.54 \AA$ ) and Bragg-Brentano geometry was used. Phase identification was done by using the FullProf [23] software. Epitaxy and thickness of the films were measured with a high-resolution X-ray diffractometer (Seifert, $\lambda=1.54 \AA$ ). To prove crystallinity of the thin films, rocking curves (for substrate off-set) and $\Theta / 2 \Theta$ measurements were carried out. For film-thickness calibration, $\mathrm{X}$-ray reflectometry curves were recorded and fitted with GenX. [24] Atomic force microscopy (AFM, Nanosurf FlexAFM, operating in tapping mode) was used to determine surface topography and roughness of the as-deposited films. AFM image processing was done using the Gwyddion [25] software.

\section{Results and discussion}

\subsection{Targets}

Qualitative phase analysis with the FullProf software revealed that all LiLSM targets contain $\mathrm{LiMn}_{2} \mathrm{O}_{4}$
[26] and $\mathrm{La}_{0.5} \mathrm{Sr}_{0.5} \mathrm{MnO}_{3}$ [27] phases except T-Li-0.33, which contains $\mathrm{LiLa}_{3} \mathrm{SrMnO} \mathrm{Mn}_{8}$ [28], $\mathrm{La}_{0.5} \mathrm{Sr}_{0.5} \mathrm{MnO}_{3}$ and $\mathrm{La}_{0.7} \mathrm{Sr}_{0.3} \mathrm{MnO}_{2.8}$. [29] Additionally all pellets contain some impurities from the starting oxides (e.g. $\mathrm{SrMnO}_{3}$ [30]).

\subsection{Thin films}

\subsubsection{Structure}

The structural analysis of the thin films grown at $923 \mathrm{~K}$ revealed a single crystalline structure (see Fig. 1a) oriented along the (h00) plane. The films exhibit the main film peak at a lower angle relative to the substrate, which is related to a larger lattice parameter. All the targets used to grow thin films for this study contained several phases. However, as it has been shown, the use of phase pure/ single crystalline targets is not strictly mandatory for PLD.

The films grown at room temperature exhibited an $\mathrm{X}$-ray amorphous structure.

X-ray reflectivity data (see Fig. 1b) were recorded to calibrate the growth rate under the same process parameters. Interestingly, films F-Li-0.33-0.15 and F-Li-0.130.01 showed an average growth of $0.125 \AA$ per laser pulse and films made from targets containing less lithium, i.e. F-Li-0.08-0.03 and F-Li-0.06-0.02 grew with 0.171 per pulse. This variation in growth rate could be due to differences in target density and therefore absorption coefficient. [32]

Height maps and surface reliefs obtained by AFM measurements for the films grown at $923 \mathrm{~K}$ are shown in Fig. 2 . The root-mean-square (RMS) surface roughness was evaluated as $1.74,2.34,1.98$ and $0.53 \mathrm{~nm}$ for rising lithium content (see Fig. 3) and a decrease in surface roughness and estimated grain size is hinted. These images indicate smooth and homogeneous films for higher lithium content. [33, 34] 


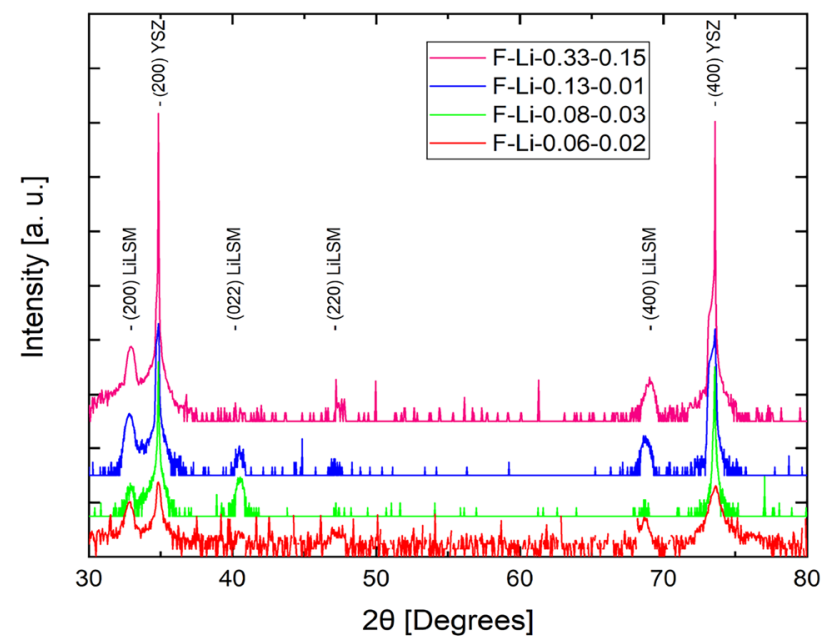

(a) X-ray diffraction pattern

Fig. 1 a X-ray diffraction patterns of thin films ablated from different targets using the same process parameters, showing the (200), (022), (200), (400) film and (200), (400) YSZ substrate orientation. The peaks for the films were indexed from [27] and the substrate peaks

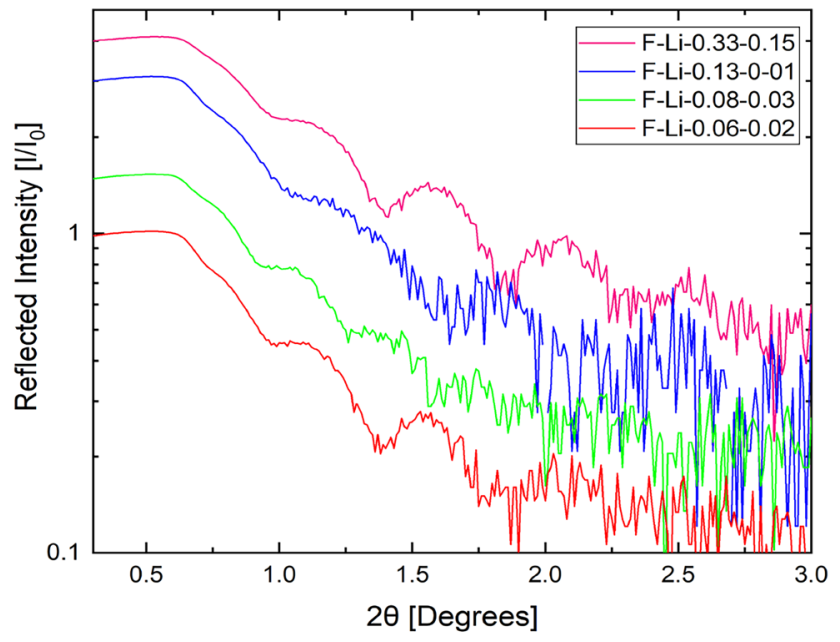

(b) Reflected intensity

from [31]. b Reflected intensity (not corrected for footprint effects) of thin films ablated from the different targets using the same process parameters each, showing different oscillation lengths and therefore thicknesses
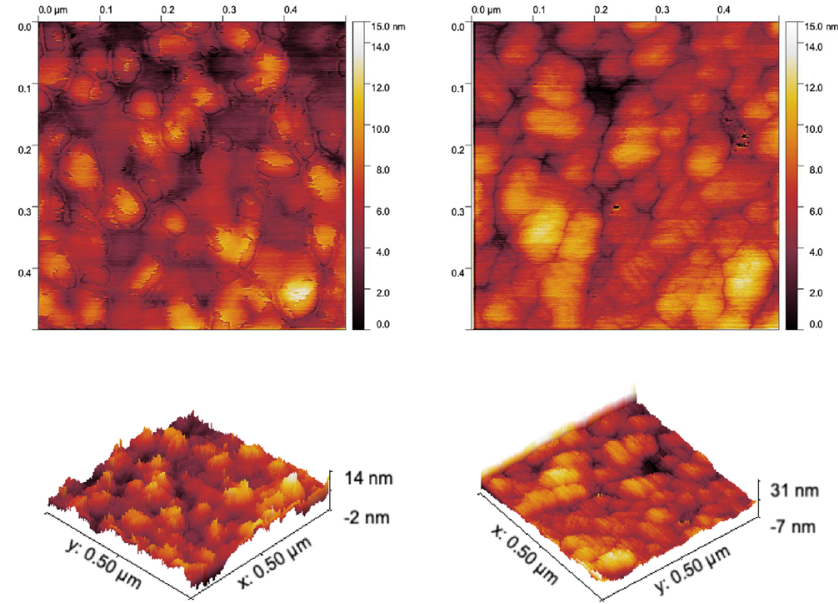

(a) F-Li-0.13-0.01

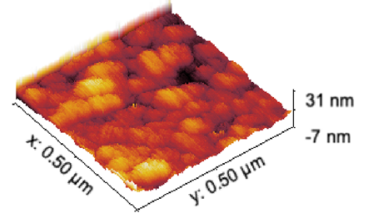

(b) F-Li-0.06-0.02
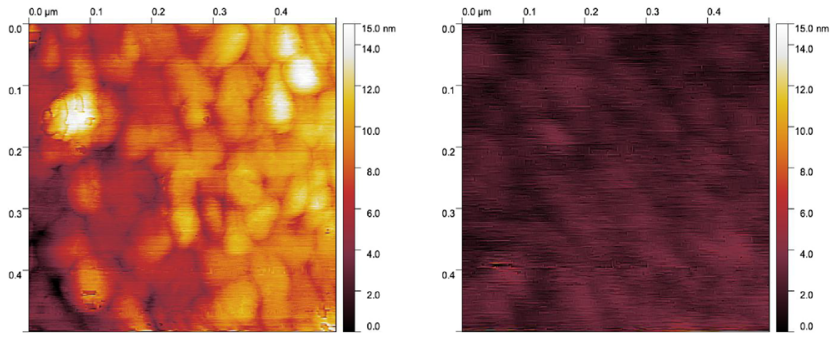

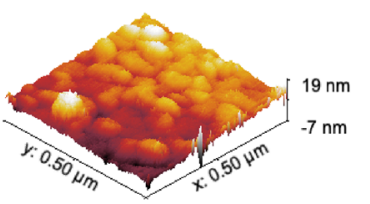

(c) F-Li-0.08-0.03

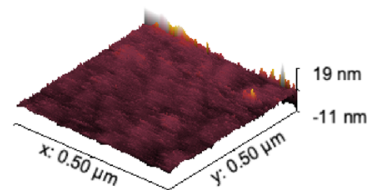

(d) F-Li-0.33-0.15

Fig. 2 Height maps and surface reliefs obtained by AFM of F-Li-0.13-0.01 a, F-Li-0.06-0.02 b, F-Li-0.08-0.03 c and F-Li-0.33-0.15 d grown at $923 \mathrm{~K}$, indicating a decrease in surface roughness and grain size for increasing lithium content

\subsubsection{Composition}

For the composition, several PLD process parameters are coming into play:

Near the target we can assume a similar average kinetic energy of the same ablated species for all targets, since their kinetic energy is related to the ablation process and the laser fluence which was kept constant at $1.5 \mathrm{~J} /$ $\mathrm{cm}^{2}$. However, the kinetic energy is also a function of the species' mass and is additionally influenced by the used background gas, pressure and interactions within the plasma plume.

The plasma plume travels to the substrate in a few $\mu$ s. Depending on the pressure and type of background gas the ablated species may encounter background gas species and interact elastically or react. The background gas (in our case $\mathrm{O}_{2}$ ) is not only used to incorporate elements into the film but also to control the kinetic energy of the arriving species. 


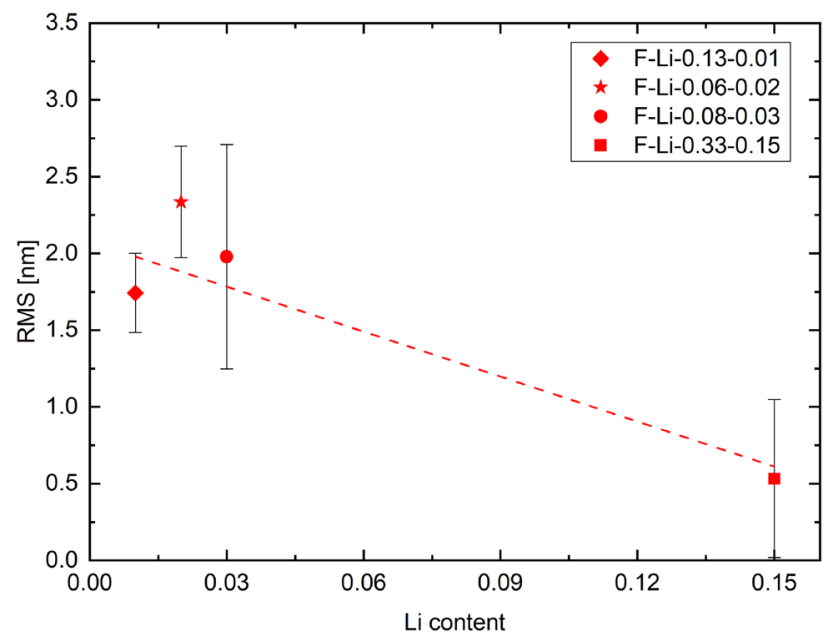

Fig. 3 Root-mean-square roughness of F-Li-0.13-0.01, F-Li-0.060.02 , F-Li-0.08-0.03 and F-Li-0.33-0.15 grown at $923 \mathrm{~K}$. The dashed line acts as a guide to the eye since the RMS for the first three films lie within their error bars

Generally, there are electrons, positive and negative ions plus neutrals in the generated plasma. These species can be excited or in ground states, additionally there can be diatomics [35] and clusters. [36] Preferentially, metals form diatomics with oxygen. The concentration of those species is proportional to their stability, meaning their bond dissociation energy, i.e. whether this is larger or smaller than the dissociation energy of oxygen of $5.2 \mathrm{eV}$. Amoruso et. al [37] classified the pressure regime $p_{\mathrm{O}_{2}}=10 \mathrm{~Pa}$ diffusion-like with a very slow expansion of the plume and a broad angular distribution of the ablated species. In the diffusion-like regime, the background gas confines all species together in a very slow expanding plume with all species travelling at a similar velocity. The mean free path (MFP) $\lambda$ of the species (see Table 3 ) is largely influenced by the pressure regime used for a given background gas. If the MFP is smaller than a tenth of the target-to-substrate distance, Amoruso et. al [37] defined this pressure regime as the transition regime which already allows chemical reactions between the background gas and the plasma plume while the plume species still have a relatively high kinetic energy when arriving at the substrate.

To achieve the formation of a crystalline LiLSM lattice, a high temperature of the substrate is needed. Heating of the substrate can have an influence on the composition and growth mode of the film. [38] In vacuum, the substrate temperature does not have a direct influence on how the species arrive at the substrate but only once they arrive. [39] However, when using a background gas the high substrate temperature heats the background gas, creating a gas density gradient which then affects the expansion dynamics of the plasma plume. This means that variations of the substrate
Table 3 MFP of the elements contained in the used targets and thin films for 10 and $10^{-3} \mathrm{~Pa}$ oxygen base pressure. Calculated according to $\lambda=\frac{k_{B} \times T}{\sqrt{2} \times P \times \pi \times d^{2}}$ with $k_{B}$ being the Boltzmann-constant, $T$ the temperature of the gas, $P$ the pressure and $d_{m}=\left(d_{O_{2}}\right)+d_{\text {species }} / 2, d$ diameter of the species

\begin{tabular}{llll}
\hline Element & Mass & $\begin{array}{l}\text { MFP in } O_{2}(10 \\
\mathrm{Pa}) \\
{[\mathrm{mm}]}\end{array}$ & $\begin{array}{l}\text { MFP in } O_{2}\left(10^{-3} \mathrm{~Pa}\right) \\
{[\mathrm{mm}]}\end{array}$ \\
\hline Lithium & 6.9 & 2.84 & $2.84 \times 10^{5}$ \\
Strontium & 87.6 & 2.06 & $2.06 \times 10^{5}$ \\
Lanthanum & 138.9 & 2.12 & $2.12 \times 10^{5}$ \\
Manganese & 54.9 & 2.93 & $2.93 \times 10^{5}$ \\
Oxygen & 16.0 & 5.28 & $5.28 \times 10^{5}$ \\
\hline
\end{tabular}

temperatures affect the amount of gas species in the path of the plume expansion and therefore varying the interaction between the plume and the background gas. Higher substrate temperatures at a given pressure will result in plume expansion dynamics typical of that at a lower pressure and vice versa. Hence, this variation of the background pressure shifts the deposition regime.

Another aspect of temperature at the substrate is re-evaporation, e.g. for $\mathrm{Li}$ at $T$ greater than $773 \mathrm{~K}$ a high loss was reported by F. Simmen. [40]

However, the most critical influence that the selected target material may have on the deposited films is their composition. For multi-element target materials, Ojeda et. al [10] reported a strong relationship between the atomic mass ratios of the target elements and the compositional deviations in the film. E.g. for $\mathrm{LiMn}_{2} \mathrm{O}_{4}$ with a mass ratio of $\mathrm{Li}$ to $\mathrm{Mn}=1: 8$, pressure dependent deviations up to $70 \%$ were observed. The deviations may be even higher if heavier elements are applied, (here $\mathrm{La}, \mathrm{Sr}$ ) with a maximum mass ratio of $\mathrm{Li}$ to $\mathrm{La}=1: 20$. During this study, deviations in lithium content of up to $90 \%$ were observed (F-Li-0.13-0.01). Lanthanum and strontium were transferred congruently.

Distance variation: For these experiments, the oxygen background pressure (10 Pa), temperature $(923 \mathrm{~K})$, laser fluence and target material (T-Li-0.13) were kept constant but the target-to-substrate distance was varied. For a distance of $4 \mathrm{~cm}$, a lithium content of 0.01 was observed. At a distance of $7 \mathrm{~cm}$, the lithium content was 0.1 . This enrichment of lithium for the larger distance could be due to the change from the diffusion-like (at $4 \mathrm{~cm}$ ) to the transition pressure regime, since the MFP (see Table 3) of the species would be below a tenth than the target-to-substrate distance as mentioned above. Since this regime allows for more chemical reactions between the species in the background gas and the formation of $\mathrm{Li}-\mathrm{O}^{+}$might be favoured on the cost of $\mathrm{Li}^{+}$in comparison to the diffusion-like regime. At a distance of 4 $\mathrm{cm}$, more $\mathrm{Li}^{+}$than $\mathrm{Li}-\mathrm{O}^{+}$species might arrive at the heated 


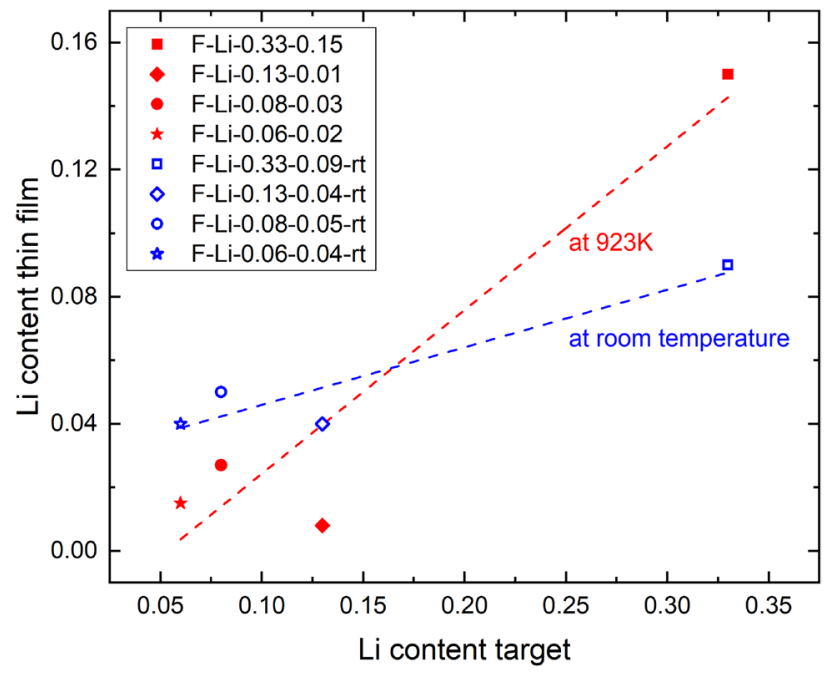

Fig. 4 Lithium content (pfu) in the targets and thin films (relative to $\mathrm{Mn}=1$ ) for ablations at $923 \mathrm{~K}$ and at room temperature, the dashed lines are guides for the eyes

substrate. Therefore, stronger lithium loss is observed, since the vapour pressure of lithium is 8 orders of magnitudes higher than for bound lithium in e.g. $\mathrm{Li}_{2} \mathrm{O}$. [41]

Temperature variation: Another series of experiments comparing compositions at $923 \mathrm{~K}$ and at room temperature (see Fig. 4) revealed that for the targets with lower lithium content, namely F-Li-0.13, F-Li-0.08, F-Li-0.06, the lithium content was higher for the room temperature ablations than for the ones at $923 \mathrm{~K}$. At room temperature, $4 \mathrm{~cm}$ targetsubstrate distance and $p_{O_{2}} \approx 10 \mathrm{~Pa}$ the pressure regime for the ablation is diffusion-like, meaning that all species are in a confined plasma plume and will arrive at the substrate at the same time. The evaporation of lithium from the unheated substrate is also much lower. Therefore, more lithium will end up in the film at room temperature compared to higher temperatures $(923 \mathrm{~K})$.

Interestingly, for target F-Li-0.33, the lithium content in the film is higher than for the room temperature ablation. This effect has to be investigated further.

After observing all these effects of parameter variation on the composition of the thin films, the right combination of target-to-substrate distance, background pressure and growth temperature was chosen to achieve the desired sample.

\section{Conclusion}

Four LiLSM PLD targets were synthesised using the solidstate route. The compositional analysis showed that one can compensate for the loss of lithium during sintering by enriching the powder with lithium before the last sintering step in agreement with literature.
Phase analysis indicated that all targets contained multiple phases while the thin films grown at $923 \mathrm{~K}$ are all single-phased.

This thin film growth study confirmed that loss of lithium growing multi-element oxides with PLD is inevitable but to some extent can be controlled by choosing the right growth conditions as temperature, background pressure, stabilised phases and especially target-to-substrate distance.

During this study, we were able to find the process parameters to grow epitaxial LiLSM thin films with the planned composition. Namely, a lithium content of 0.10 by using a target with $30 \%$ excess lithium (relative to the aimed for composition), a substrate temperature of $923 \mathrm{~K}$, a target-to-substrate distance of $7 \mathrm{~cm}$ and an oxygen background pressure of $20 \mathrm{~Pa}$. Further studies investigating magnetic and other physical properties will be conducted with these samples.

Acknowledgements This project is funded by the Swiss National Science Foundation under grant: 200021_169704 and carried out at Paul Scherrer Institute, Switzerland. The ICP-MS measurements were done in collaboration with Institute of Chemical Technologies and Analytics, TU Wien, Austria. L. Indrizzi for technical assistance.

Funding Open Access funding provided by Lib4RI - Library for the Research Institutes within the ETH Domain: Eawag, Empa, PSI \& WSL.

\section{Declaration}

Conflict of interest The authors declare that they have no conflict of interest.

Open Access This article is licensed under a Creative Commons Attribution 4.0 International License, which permits use, sharing, adaptation, distribution and reproduction in any medium or format, as long as you give appropriate credit to the original author(s) and the source, provide a link to the Creative Commons licence, and indicate if changes were made. The images or other third party material in this article are included in the article's Creative Commons licence, unless indicated otherwise in a credit line to the material. If material is not included in the article's Creative Commons licence and your intended use is not permitted by statutory regulation or exceeds the permitted use, you will need to obtain permission directly from the copyright holder. To view a copy of this licence, visit http://creativecommons.org/licenses/by/4.0/.

\section{References}

1. F.K. Shokoohi, J.M. Tarascon, B.J. Wilkens, Fabrication of thinfilm $\mathrm{LiMn}_{2} \mathrm{O}_{4}$ cathodes for rechargeable microbatteries. Appl. Phys. Lett. 59, 1260-1262 (1991). https://doi.org/10.1063/1. 105470

2. A. Manthiram, X. Yu, and S. Wang. Lithium battery chemistries enabled by solid-state electrolytes. Nat. Rev. Mat., 2, 2017. https://doi.org/10.1038/natrevmats.2016.103 
3. B. Yan, J. Liu, and B. et al. 2013 Song. Li-rich thin film cathode prepared by pulsed laser deposition. Sci. Rep., 3:3332, https:// doi.org/10.1038/srep03332

4. N. Kuwata, R. Kumar, K. Toribami, T. Suzuki, T. Hattori, J. Kawamura, Thin film lithium ion batteries prepared only by pulsed laser deposition. Solid State Ionics 177, 2827-2832 (2006). https://doi.org/10.1016/j.ssi.2006.07.023

5. W. Pfleging, A review of laser electrode processing for development and manufacturing of lithium ion batteries. Nanophotonics 7(3), 549-573 (2018). https://doi.org/10.1515/ nanoph-2017-0044

6. J.L. Ortiz-Quiñonez, García-González L., Cancino-Gordillo F.E., and Pal U. 2020 Particle dispersion and lattice distortion induced magnetic behavior of $\mathrm{La}_{1-x} \mathrm{Sr}_{x} \mathrm{MnO}_{3}$ perovskite nanoparticles grown by salt-assisted solid-state synthesis. Mat. Chem. Phys., 246:122834. https://doi.org/10.1016/j.matchemphys.2020.122834

7. S. Dasgupta, B. Das, M. Knapp, R.A. Brand, H. Ehrenberg, R. Kruk, H. Hahn, Intercalation- driven reversible control of magnetism in bulk ferromagnets. Adv. Mat. 26, 4639-4644 (2014). https://doi.org/10.1002/adma.201305932

8. T. Venkatesan, (2013) Pulsed laser deposition - invention or discovery? J. Phys. D: Appl. Phys. https://doi.org/10.1088/00223727/47/3/034001

9. J. Schou, Physical aspects of the pulsed laser deposition technique: the stoichiometric transfer of material from target to film. Appl. Surf. Sci. 255, 5191-5198 (2009). https://doi.org/10.1016/j.apsusc. 2008.10.101

10. A. Ojeda, C.W. Schneider, M. Döbeli, T. Lippert, The importance of pressure and mass ratios when depositing multi-element oxide thin films by pulsed laser deposition. Appl. Surf. Sci. 389, 126-134 (2016). https://doi.org/10.1016/j.apsusc.2016.07.003

11. I. Marozau, A. Shkabko, G. Dinescu, M. Döbeli, T. Lippert, D. Logvinovich, M. Mallepell, C.W. Schneider, A. Weidenkaff, A. Wokaun, Pulsed laser deposition and characterisation of nitrogensubstituted $\mathrm{SrTiO}_{3}$ thin films. Appl. Surf. Sci. 255, 5252-5255 (2009). https://doi.org/10.1016/j.apsusc.2008.07.159

12. D.B. Chrisey, G.K. Hubler, Pulsed Laser Deposition of Thin Films (Wiley, New York, 1994)

13. J. Chen, M. Döbeli, D. Stender, K. Conder, A. Wokaun, C.W. Schneider, T. Lippert, Plasma interactions determine the composition in pulsed laser deposited thin films. Appl. Phys. Lett. 105, 1-5 (2014). https://doi.org/10.1063/1.4895788

14. A. Ojeda, M. Döbeli, T. Lippert, Influence of plume properties on thin film composition in pulsed laser deposition. Adv. Mat. Int. 5, 1-26 (2018). https://doi.org/10.1002/admi.201701062

15. T. Ohnishi, B.T. Hang, X. Xu, M. Osada, K. Takada, Quality control of epitaxial $\mathrm{LiCoO}_{2}$ thin films grown by pulsed laser deposition. J. Mater. Res. 25, 1886-1889 (2010). https://doi.org/10.1557/jmr.2010.0250

16. T. Dumont, T. Lippert, M. Döbeli, H. Grimmer, J. Ufheil, P. Novák, A. Würsig, U. Vogt, A. Wokaun, Influence of experimental parameters on the Li-content of $\mathrm{LiMn}_{2} \mathrm{O}_{4}$ electrodes produced by pulsed laser deposition. Appl. Surf. Sci. 252, 4902-4906 (2006). https:// doi.org/10.1016/j.apsusc.2005.07.119

17. A.J. Millis, Lattice effects in magnetoresistive manganese perovskites. Nature 392, 147-150 (1998). https://doi.org/10.1038/32348

18. M.B. Salamon, M. Jaime, The physics of manganites: structure and transport. Rev. Mod. Phys. 73, 583-628 (2001). https://doi.org/10. 1088/0022-3727/38/8/R01

19. G. Vasta, A. Feteira, D.I. Woodward, D. Walker, P.A. Thomas, T.J. Jackson, Thin film $\mathrm{LaYbO}_{3}$ capacitive structures grown by pulsed laser deposition. Thin Solid Films 527, 81-86 (2013). https://doi. org/10.1016/j.tsf.2012.12.006

20. D. Mandal, M.C. Jadeja, N.S. Ghuge, D. Sen, S. Mazumder, Effect of excess lithium on sintering behaviour of lithium-titanate pebbles: modifications of microstructure and pore morphology. Fusion Eng. Des. 112, 520-526 (2016). https://doi.org/10.1016/j.fusengdes.2016.05.041
21. M. P. de Jong, I. Bergenti, V. A. Dediu, M. Fahlman, M. Marsi, and C. Taliani. 2005 Evidence for $\mathrm{Mn}^{2+}$ ions at surfaces of $\mathrm{La}_{0.7} \mathrm{Sr}_{0.3} \mathrm{MnO}_{3}$ thin films. Phys. Rev. B. 71(014434), . https://doi. org/10.1103/PhysRevB.71.014434

22. S.F. Boulyga, H.J. Dietze, S.J. Becker, Determination of the stoichiometry and trace impurities in thin barium strontium titanate perovskite layers by inductively coupled plasma-mass spectrometry. J. Anal. At. Spectrom. 16, 598-602 (2001). https://doi.org/10.1039/B101339O

23. J. Rodrigez-Carvajal. Fullprof: a program for rietveld refinement and pattern matching analysis. Abstracts of the Satellite Meeting on Powder Diffraction of the XV Congress of the IUCr, page 127, 1990

24. M. Bjoerck, G. Andersson, Genx: an extensible x-ray reflectivity refinement program utilizing differential evolution. J. Appl. Cryst. 40, 1174 (2007). https://doi.org/10.1107/S0021889807045086

25. an open-source software for spm data analysis, D. Neč as and P. Klapetek. Gwyddion. Central European J. Phys. 10, 181-188 (2012). https://doi.org/10.2478/s11534-011-0096-2

26. H. Berg, J.O. Thomas, L. Wen, G.C. Farrington, A neutron diffraction study of ni substituted $\mathrm{LiMn}_{2} \mathrm{O}_{4}$. Solid State Ionics 112, 165-168 (1998). https://doi.org/10.1016/S0167-2738(98)00167-2

27. M. Hazzez, N. Ihzaz, M. Boudard, and M. Oumezzine. 2015 The structural, magnetic and above room temperature magnetocaloric properties of $\mathrm{La}_{05} \mathrm{Sr}_{05} \mathrm{MnO}_{3}$ compound. European Phys. J. Plus, 130: 1-8, . https://doi.org/10.1140/epjp/i2015-15179-0

28. J.C. Burley, P.D. Battle, D.J. Gallon, J. Sloan, C.P. Grey, M.J. Rosseinsky, Magnetism and structural chemistry of the $\mathrm{n}=1$ ruddlesden-popper phases $\mathrm{La}_{4} \mathrm{LiMnO}$ and $\mathrm{La}_{3} \mathrm{SrLiMnO}$. J. Am. Chem. Soc. 124, 620-628 (2002). https://doi.org/10.1021/ja012023z

29. S.V. Trukhanov, I.O. Troyanchuk, I.A. Bobrikov, V.G. Simkin, and A.M. Balagurov. 2007 Structure phase separation in the aniondeficient $\mathrm{La}_{0.7} \mathrm{Sr}_{0.3} \mathrm{MnO}_{3-d}$ manganite system. Poverkhnostnye Fizika, Khimiya, Mekhanika, 12:11-17, https://doi.org/10.1134/ S1063774507050094

30. K. Kuroda, N. Ishizawa, N. Mizutani, M. Kato, The crystal structure of alpha-SrMnO${ }_{3}$. J. Solid State Chem. 38, 297-299 (1981). https:// doi.org/10.1016/0022-4596(81)90059-1

31. M. Ando, I. Oikawa, Y. Noda, S. Ohki, M. Tansho, T. Shimizu, H. Kiyono, H. Maekawa, High-field o-17 nmr study of defects in doped zirconia and ceria. Solid State Ionics 192, 576-579 (2011). https:// doi.org/10.1016/j.ssi.2010.04.024

32. S.N. Ogugua, O.M. Ntwaeaborwa, H.C. Swart, Latest development on pulsed laser deposited thin films for advanced luminescence applications. Coatings 10(1078), 583-628 (2020)

33. S.B. Tang, M.O. Lai, L. Lu, Electrochemical studies of low-temperature processed nano-crystalline $\mathrm{LiMn}_{2} \mathrm{O}_{4}$ thin film cathode at 55c. J. Power Sour. 164, 372-378 (2007). https://doi.org/10.1016/j. jpowsour.2006.09.109

34. M. Salah, S. Azizi, C. Khaldi, and J. Lamloumi.2018 Nanoindentation and afm characterization of lithium-doped zno sprayed thin films. 2018 9th International Renewable Energy Congress (IREC), https://doi.org/10.1109/IREC.2018.8362567

35. M. Esposito, M. Bator, M. Döbeli, T. Lippert, C. Schneider, A. Wokaun, Negative ions: the overlooked species in thin film growth by pulsed laser deposition. Appl. Phys. Lett. 99, 19150 (2011). https://doi.org/10.1063/1.3660399

36. W. Marine, L. Patrone, B. Luk'yanchuk, and Sentis M. 2000 Strategy of nanocluster and nanostructure synthesis by conventional pulsed laser ablation. Appl. Surf. Sci, 154:345 .https://doi.org/10. 1016/S0169-4332(99)00450-X

37. S. Amoruso, B. Toftmann, J. Schou, Thermalization of a uv laser ablation plume in a background gas: From a directed to a diffusionlike flow. Phys. Rev. E 69, 056403 (2004). https://doi.org/10.1103/ PhysRevE.69.056403

38. A. Ojeda, C.W. Schneider, T. Lippert, A. Wokaun, Pressure and temperature dependence of the laser-induced plasma plume 
dynamics. Appl. Phys. 120, 225301 (2016). https://doi.org/10. $1063 / 1.4971251$

39. A. Sambri, S. Amoruso, X. Wang, M. Radovic, F. Miletto Granozio, and Buzzese R. Substrate 2007 heating influence on plume propagation during pulsed laser deposition of complex oxides. Appl.Phys. Lett. 91:151501, https://doi.org/10.1063/1.2795792

40. F. Simmen. Pulsed Laser Deposition zur Herstellung von Modellphasengrenzen für elektrochemische Untersuchungen. ETH Zürich, 2010

41. J.M. Hur, S.M. Jeong, H.S. Lee, Status of pyroprocessing technology development in Korea. Nucl. Eng. Technol. 42, 73 (2010). https://doi.org/10.5516/NET.2010.42.2.131
Publisher's Note Springer Nature remains neutral with regard to jurisdictional claims in published maps and institutional affiliations. 